\title{
Tailoring Passivation Molecular Structures for Extremely Small Open-Circuit Voltage Loss in Perovskite Solar Cells
}

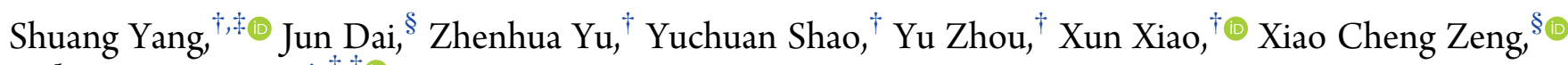 \\ and Jinsong Huang*,
}

\author{
${ }^{\dagger}$ Department of Applied Physical Sciences, University of North Carolina, Chapel Hill, North Carolina 27599, United States \\ ${ }^{\ddagger}$ Department of Mechanical and Materials Engineering and ${ }^{\S}$ Department of Chemistry, University of Nebraska-Lincoln, Lincoln, \\ Nebraska 68588, United States
}

\begin{abstract}
Passivation of electronic defects at the surface and grain boundaries of perovskite materials has become one of the most important strategies to suppress charge recombination in both polycrystalline and singlecrystalline perovskite solar cells. Although many passivation molecules have been reported, it remains very unclear regarding the passivation mechanisms of various functional groups. Here, we systematically engineer the structures of passivation molecular functional groups, including carboxyl, amine, isopropyl, phenethyl, and tert-butylphenethyl groups, and study their passivation capability to perovskites. It reveals the carboxyl and amine groups would heal charged defects via electrostatic interactions, and the neutral iodine related defects can be reduced by the aromatic structures. The judicious control of the interaction between perovskite and molecules can further realize grain boundary passivation, including those that are deep toward substrates. Understanding of the underlining mechanisms allows us to design a new passivation molecule, D-4-tert-butylphenylalanine, yielding
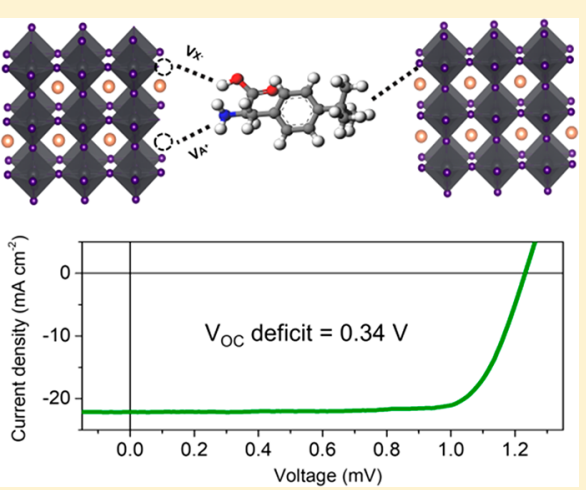
high-performance $\mathrm{p}$-i-structure solar cells with a stabilized efficiency of $21.4 \%$. The open-circuit voltage $\left(V_{\mathrm{OC}}\right)$ of a device with an optical bandgap of $1.57 \mathrm{eV}$ for the perovskite layer reaches $1.23 \mathrm{~V}$, corresponding to a record small $V_{\mathrm{OC}}$ deficit of $0.34 \mathrm{~V}$. Our findings provide a guidance for future design of new passivation molecules to realize multiple facets applications in perovskite electronics.
\end{abstract}

\section{INTRODUCTION}

Halide perovskite solar cells (PSCs) are one of the most promising next-generation photovoltaic technologies that can potentially drive down the cost of clean and renewable solar energy conversion. The achieved power conversion efficiencies (PCEs) are already over $24 \%$ for the solution-processed PSCs which are low-cost and can be scaled up. ${ }^{1}$ The polycrystalline perovskite films in the state-of-the-art PSCs have a large number of defects, including amorphous regions, surface defects, and grain boundaries, which are sensitive to material process. ${ }^{2-6}$ Though these materials are reported to have more benign defects compared to other semiconductors, ${ }^{7}$ the defects at film surface and grain boundaries still induce a high density of trap states which dramatically impair the efficiency and stability of PSCs. ${ }^{8}$ A faster photoluminescence decay at grain boundaries or unpassivated perovskite film surfaces is indicative of fast nonradiative charge recombination and the electronically defective nature of grain boundaries and film surfaces. ${ }^{9}$

Like all other inorganic PV materials, passivation of electronic surface trap states has been shown to be also necessary to reduce the charge recombination rate and enhance the efficiency of PSCs. ${ }^{10,11}$ However, this was not obvious to the society when these materials were initially treated as organic dyes. The passivation concept was first introduced by us in p-i-n structured PSCs with fullerene as the electron transport layer. ${ }^{12,13}$ Since then, a number of passivation molecules have been reported to directly neutralize the surface charges or dangling bonds to annihilate the corresponding electronic traps. For example, the undercoordinated $\mathrm{Pb}$ ions can be passivated by bonding with Lewis base molecules which can donate electrons or share their electron pairs, such as $n$-trioctylphosphine oxide. ${ }^{14}$ In addition, some electronic trap states can be reduced by Lewis acid molecules by acceptation of one electron from the Lewis based type defects on perovskite surface, such as phenyl-C61-butyric acid methyl ester (PCBM). ${ }^{12,13}$ The surface charged sites can also be neutralized by charged molecules or ions, such as positively charged phenethylammonium, negatively charged chloride ion, and choline chloride, which is a zwitterion. ${ }^{10,15,16}$ Although numerous approaches have been employed, the well passivation of surface electronic defects at grain boundaries still remains a great challenge because of the complexity and diversity of the surface defects that most of the electronic materials can only passivate one or two kinds of trap states. It

Received: December 14, 2018

Published: March 19, 2019 
is very unclear so far what functional groups are playing the passivation roles among passivation molecules with complicated chemical structures. Another critical issue is that most of the passivation methods are based on the surface coating or treatment of the top surface, which cannot effectively passivate the traps from inner grain boundaries toward the substrates within the perovskite films.

In this work, we systematically study the influence of molecular structure of passivation molecules on their passivation effect to hybrid perovskites. It is found that some small sized passivation molecules can spontaneously distribute at grain boundaries, which dramatically enhance the defect passivation at grain boundaries of hybrid perovskites. Through the analysis, we discovered a new type of amino acid passivation molecule, D-4-tert-butylphenylalanine (D4TBP), which combines all effective passivation groups and most efficiently passivate perovskite defects. As a proof of concept, we show that D4TBP passivated p-i-n solar cell (Figure 1a) has a record low $V_{\mathrm{OC}}$ loss $\left(E_{\mathrm{g}} / q-V_{\mathrm{OC}}, E_{\mathrm{g}}\right.$ is the bandgap of perovskite) of only $0.34 \mathrm{eV}$.

\section{RESULTS AND DISCUSSION}

The ionic nature of hybrid perovskites determines that their defects are charged. The defects with negative or positive charges illustrated in Figure $1 \mathrm{~b}$ have been experimentally and theoretically studied. ${ }^{17,18}$ These surface charged species typically provide extra energy levels within the forbidden gap
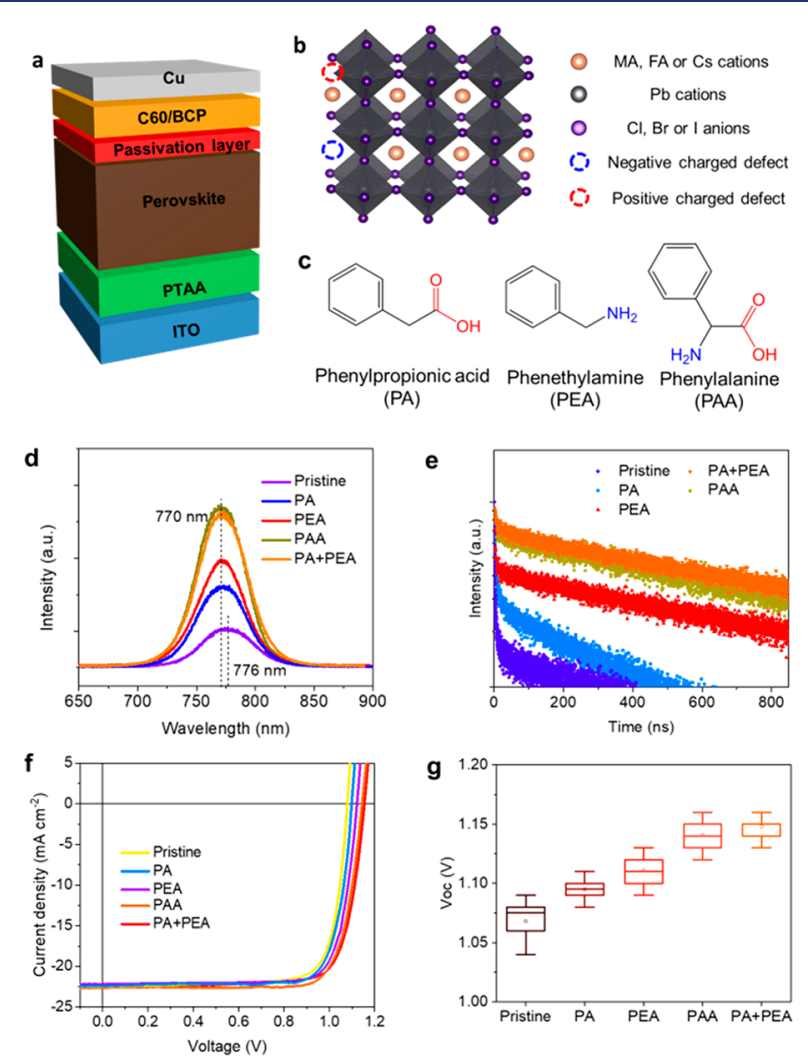

Figure 1. (a) Device structure of planar heterojunction perovskite solar cells. (b) Schematic illustration of surface charged defects. (c) Chemical structure of passivation molecules with marked amino (blue) and carboxyl (red) groups. (d) PL and (e) TRPL spectra of perovskite films with different passivation layers. (f) $J-V$ curves and (g) statistics of $V_{\mathrm{OC}}$ distribution of perovskite solar cells with different passivation layers. which behave as charge traps. We first study whether one type of defect, positively charged or negatively charged, dominates on the perovskite surface and whether passivation of one of them is more effective in enhancing device efficiency. To neutralize these charged defects, phenylpropionic acid (PA) with a carboxyl group and phenethylamine (PEA) with an amine group were first used, which are expected to combine with positively and negatively charged defects, respectively (Figure 1c). Phenylalanine (PAA) with both carboxyl and amine groups was also chosen in this study and may passivate both negatively and positively charged defects. All passivation layers were formed by spin-coating of solution of the passivation molecules on the surfaces of perovskite films (see details in the Experimental Section). This avoids the complication induced by perovskite morphology changes in the case of mixing the additives in the perovskite solution, though mixing them directly is preferred in fabrication to reduce one step. It should be noted that passivation molecules may also penetrate into the polycrystalline films along the grain boundaries after spin-coating as observed in our previous results. $^{12}$

To assess the passivation effect of different molecules, we measured the steady-state photoluminescence (PL) and timeresolved photoluminescence (TRPL) decay of the films and current density-voltage $(J-V)$ characteristics of the devices with different passivation layers. Triple-cation perovskite with a chemical formula of $\mathrm{Cs}_{0.05} \mathrm{FA}_{0.81} \mathrm{MA}_{0.14} \mathrm{PbI}_{2.55} \mathrm{Br}_{0.45}$ in precursors was employed in this study. An excitation light of $532 \mathrm{~nm}$ was used for the steady-state PL measurement to probe the surface properties, which has a penetration length of $80 \mathrm{~nm}$, much less than the thickness of the perovskite films $(\sim 500$ $\mathrm{nm}$ ). As shown in Figure 1d, the PL intensity of PA and PEA films is about 2.3 and 2.9 times larger compared with that of the pristine one, indicating that the carboxyl and amine groups could passivate defects with specific charges. The PL decay of the perovskite films in Figure 1e shows biexponential decays with a fast and a slow component. The short lifetime $\tau_{1}$ is a decay component related to the charge trapping process and the long lifetime $\tau_{2}$ as a component of the detrapping process or carrier recombination process. ${ }^{19}$ The PL decay of pristine film without any passivation is dominated by the charge trapping process; i.e., over $90 \%$ of excess charge carriers are trapped immediately after generation. The $\tau_{1}$ of perovskite films increases from $1.51 \mathrm{~ns}$ to 4.42 and $4.24 \mathrm{~ns}$ upon the passivation of PA and PEA, respectively (Table S1). In addition, the PL decay in the PA and PEA treated samples is dominated by the long decay process, implying charge trapping in PA and PEA films is suppressed. Amines and carboxylic acids have been widely used as the ligands in the synthesis of perovskite nanocrystals, which can adsorb onto different surface sites and generate high PL quantum yields over $80 \% .^{20,21}$ Previous literatures have also demonstrated the good passivation effect of PEA molecules in solar cells. ${ }^{14,23}$ In solution, amino or carboxyl groups readily ionize with positive and negative charges, respectively; we speculate that they may passivate different types of defects present on perovskite film surface, and thus combination of them would synergistically passivate more defects. To verify this, we used the PA and PEA mixture $(\mathrm{PA}+\mathrm{PEA})$ as the passivation layer. As expected, the perovskite films treated by a PA-PEA mixture have shown a much larger PL intensity and longer PL lifetimes $\left(\tau_{1}=12.50\right.$ $\left.\mathrm{ns}, \tau_{2}=780.61 \mathrm{~ns}\right)$, which confirms the synergetic passivation effect among these two passivation function groups on 
different defect sites on perovskites. We move a future step to use a single molecule, PAA, to replace the PA-PEA mixture in passivation because PAA has the combination of phenethyl, amine, and carboxyl functional groups. As expected, PAA passivated perovskite films also benefit from the passivation effect of both functional groups, as evidenced by the strong, blue-shifted PL signal compared with PA+PEA sample and prolonged $\tau_{1}$ and $\tau_{2}$ of 9.80 and 737.33 ns, respectively, comparable to those of the sample treated by the PA-PEA mixture.

The passivation of defects should considerably impact the open circuit voltage $\left(V_{\mathrm{OC}}\right)$ of the devices due to their enhanced carrier concentration under light irradiation by uplifting the quasi-Fermi level splitting. To evaluate it, $p$-i-n structure solar cells were fabricated with schematic structure of poly[bis(4-phenyl) (2,4,6-trimethylphenyl)amine](PTAA)/ perovskite/passivation layer/fullerene $\left(\mathrm{C}_{60}\right) / 2,9$-dimethyl-4,7diphenyl-1,10-phenanthroline $(\mathrm{BCP}) /$ copper $(\mathrm{Cu})$, as shown in Figure 1a. The pristine champion device shows a shortcircuit current density $\left(J_{\mathrm{SC}}\right)$ of $22.40 \mathrm{~mA} \mathrm{~cm}{ }^{-2}$, a $V_{\mathrm{OC}}$ of 1.08 $\mathrm{V}$, a fill factor (FF) of 0.788 , and a higher PCE of $19.1 \%$ (Figure 1f). Solar cells based on PA and PEA layers exhibited improved average $V_{\mathrm{OC}}$ of 1.10 and $1.11 \mathrm{~V}$ and PCE of 19.0 and $19.2 \%$, respectively, compared to that of the pristine device (Figure $1 \mathrm{~g}$ and Table S2). The reduction of surface defects will lead to higher density of photogenerated charges under given illumination and therefore provide larger quasi-Fermi level splitting and $V_{\mathrm{OC}}$ values. Mixing PA and PEA gives a similar passivation effect with PAA, which is consistent with PL and TRPL studies and again showing the synergetic passivation of the functional groups. A high average $V_{\mathrm{OC}}$ of $1.15 \mathrm{~V}$ was achieved in the devices with mixed PA and PEA additives. When we used PAA with both carboxyl and amino groups as the passivation layer, the champion device delivered a $J_{S C}$ of $22.62 \mathrm{~mA} \mathrm{~cm}^{-2}$, a $V_{\mathrm{OC}}$ of $1.15 \mathrm{~V}$, a FF of 0.790 , and a higher PCE of $20.6 \%$ (Figure 1f). The average $V_{\mathrm{OC}}$ of PAA devices is $1.14 \mathrm{~V}$, which is consistent with the PL results.

In addition to the charged molecules, functional groups with neutral and nonpolar structures, such as fullerene or conjugated organics, also have passivation capability through the coordination interactions with perovskite surface. ${ }^{13}$ Previous X-ray photoelectron spectroscopy (XPS) studies have revealed the existence of neutral species, such as unsaturated $\mathrm{Pb}^{0}$ which appeared on the surface of hybrid perovskite films, because of the oxidation of $\mathrm{I}^{-}$into $\mathrm{I}_{2}{ }^{23-25}$ Moreover, the existence of residual iodine will introduce deep trap states by the formation of I interstitials or $\mathrm{I}-\mathrm{Pb}$ antisites according to the previous theoretical study. ${ }^{26,27}$ Zhang et al. further validated the electronically acceptor nature of these iodine-related defects by surface photovoltage (SPV) measurements. ${ }^{28}$ We thus speculate that an electron-donating aromatic group may reduce the acceptor type trap states induced by dissociative iodine. A PAA molecule has six $\pi$ electrons delocalized in the aromatic benzene ring which may act as Lewis base to form Lewis adducts with specific Lewis-type defects. Here, we compared three amino acid molecules, valine (VA), PAA with isopropyl, phenyl, and tert-butylphenethyl groups, respectively, whose chemical structures are shown in Figure 2a. By comparison of the passivation effect of VA and PAA treated films, the function of aromatic rings can be determined because the only difference of PAA and VA is the presence of an aromatic ring in PAA. a
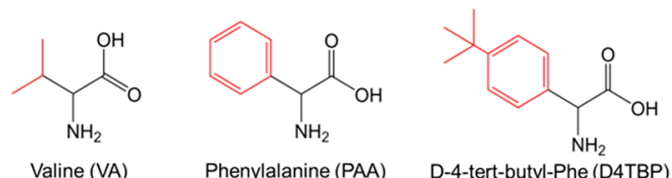

b
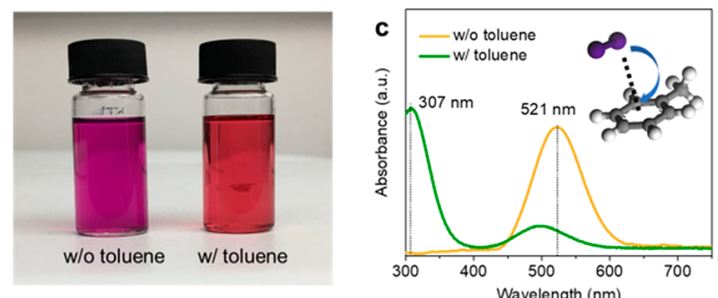

d
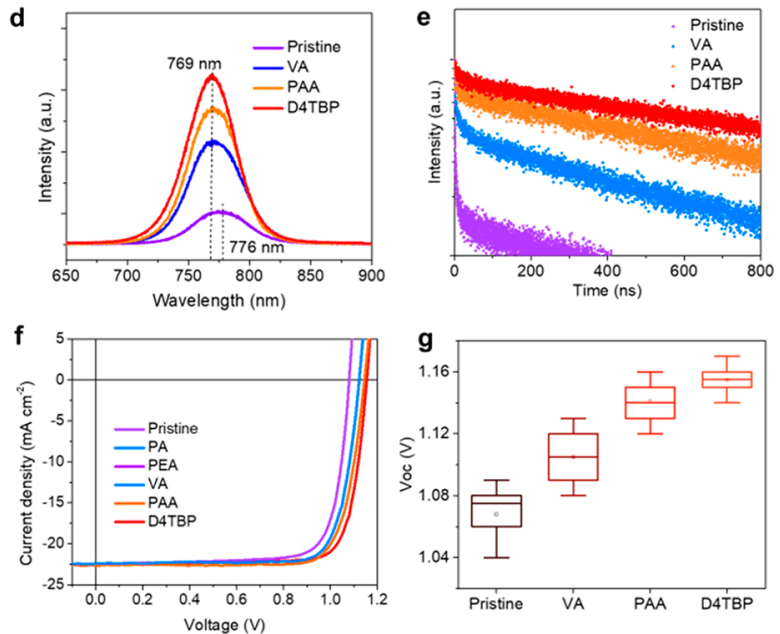

Figure 2. (a) Chemical structure of passivation molecules with marked alkyl and phenyl groups. (b) Photograph of iodine dissolved in the hexane solution without and with $20 \%$ toluene (v:v). The concentration of iodine is $1 \mathrm{mg} / \mathrm{mL}$. (c) UV-vis absorption spectra of iodine dissolved in different solvents. (d) PL and (e) TRPL spectra of perovskite films with different passivation layers. (f) $J-V$ curves and $(\mathrm{g})$ statistics of $V_{\mathrm{OC}}$ distribution of perovskite solar cells with different passivation layers.

We used $\mathrm{I}_{2}$-toluene to explore the possible interaction of $\mathrm{I}_{2}$ and benzene rings. The iodine-hexane solution has a purple color, as shown in Figure $2 \mathrm{~b}$, which is the same color with solid iodine. When we added $20 \mathrm{vol} \%$ toluene in the solution, the color of the iodine solution became dark red. As shown in Figure $2 \mathrm{c}$, the absorption spectrum of this mixed solution shows the emergence of an absorption peak around $307 \mathrm{~nm}$, which suggests the formation of benzene-iodine complexes as a result of the electron-donating property of the benzene ring. ${ }^{29}$ Thus, aromatic rings could reduce or capture the trace amount of $\mathrm{I}_{2}$ existing as electronic defects in perovskites. This is supported by the steady-state and transient PL study. Although the PL intensity of VA is enhanced to 3.2 times larger than pristine sample, it is still lower than the PAA sample ( $\sim 4.4$ times), which implies the phenethyl group is also involved in surface passivation (Figure $2 \mathrm{~d}$ ). Moreover, the PL decay process in the PAA passivated films is much slower than in VA passivated films, as shown in Figure 2e, implying that aromatic structure reduced the charge capture rate by deep traps, most likely due to reduced density of excess-iodineinduced electronic trap states at the surface.

On the basis of the understanding of passivation mechanism, we design a new passivation molecule D4TBP. The chemical 
structure in Figure 2a shows that it contains all three passivation functional groups, i.e., 4-tert-butylphenyl, amine, and carboxyl ones. When D4TBP was used, the charge recombination lifetime of perovskite films is increased to $930.30 \mathrm{~ns}$, and the charge-trapping process becomes negligible. There is one more tert-butyl group on the benzene ring in D4TBP compared with the PAA molecule, which may strengthen the electron-donating capability of the benzene ring and thus better passivate Lewis acid type surface defects, such as iodine and undercoordinated $\mathrm{Pb}^{2+}$. Figure $2 \mathrm{f}$ shows the $J-V$ characteristics without and with different passivation layers. The champion device with the D4TBP passivating layer exhibits excellent performance with a $J_{\mathrm{SC}}$ of $22.51 \mathrm{~mA} \mathrm{~cm}{ }^{-2}$, a $V_{\mathrm{OC}}$ of $1.16 \mathrm{~V}$, and a FF of 0.803 , yielding a PCE of $21.0 \%$, greatly outperforming the control cell. As exhibited in Figure $2 \mathrm{~g}$, the average $V_{\mathrm{OC}}$ of VA, PAA, and D4TBP is $1.11,1.14$, and $1.16 \mathrm{~V}$, respectively, which again agree with the PL and TRPL results.

The aforementioned results have demonstrated the molecular engineering strategy to reduce the surface electronic defects. We then focus on another question: how to more effectively passivate the grain boundaries in polycrystalline perovskite films in addition to the top surfaces. If the passivation molecules are used as additive, they could be adsorbed onto the surfaces as well as the grain boundaries during the crystallization and growth process, which may enable a full coverage of grains. Here we added around $7.5 \mathrm{mM}$ of additives into perovskite solution and evaluated their passivation function. These additives do not cause the formation of impure phases, as shown in the X-ray diffraction (XRD) pattern in Figure S1. We do understand these additives or ligands would hinder grain growth and thus reduce the grain size, if they strongly bind to the crystal surface. Actually, when PA and PEA are added, the grain size of the resulting perovskite films reduces obviously from $312.0 \pm 52.9 \mathrm{~nm}$ to be $138.7 \pm 33.9$ and $99.3 \pm 27.8 \mathrm{~nm}$, respectively (Figure 3a and Figure S2). The grain sizes of VA, PAA, and D4TBP samples are $284.8 \pm 57.4,267.3 \pm 49.0$, and $287.1 \pm 46.2 \mathrm{~nm}$, respectively, as shown in Figure $3 \mathrm{~b}$. We speculate the amine group in amino acids may not participate into the perovskite crystal due to the steric effect of the large molecules. ${ }^{30}$ Nevertheless, amino acids should be excluded from perovskite crystals to grain boundary areas or interfaces, and passivation can be realized by reasonably tuning the adsorption and desorption behavior of passivation molecules without significantly reducing grain size. Here we performed PL measurement to identify the position of D4TBP molecules when incorporated as additive, and the setup of the experiment is shown in Figure 3c. The spontaneous radiative recombination via trap states typically resulted a red-shifted emission compared with that from the band-edge transition. Passivation of these trap states can blue-shift the PL peak. As shown in Figure 3d, the perovskite film with a D4TBP layer displayed a strong PL peak at $769 \mathrm{~nm}$ at the top side, whereas the PL emission at the glass side red-shifted to $775 \mathrm{~nm}$ with a much weaker signal. This suggests the surface defects are effectively passivated by the D4TBP layer on only the top side by the spin-coating method. However, the PL peak was independent of the incident light directions for the films passivated by the additive route, illustrating the top and bottom surfaces have similar optical properties. Therefore, it is believed that the D4TBP would be spontaneously excluded and distributed at
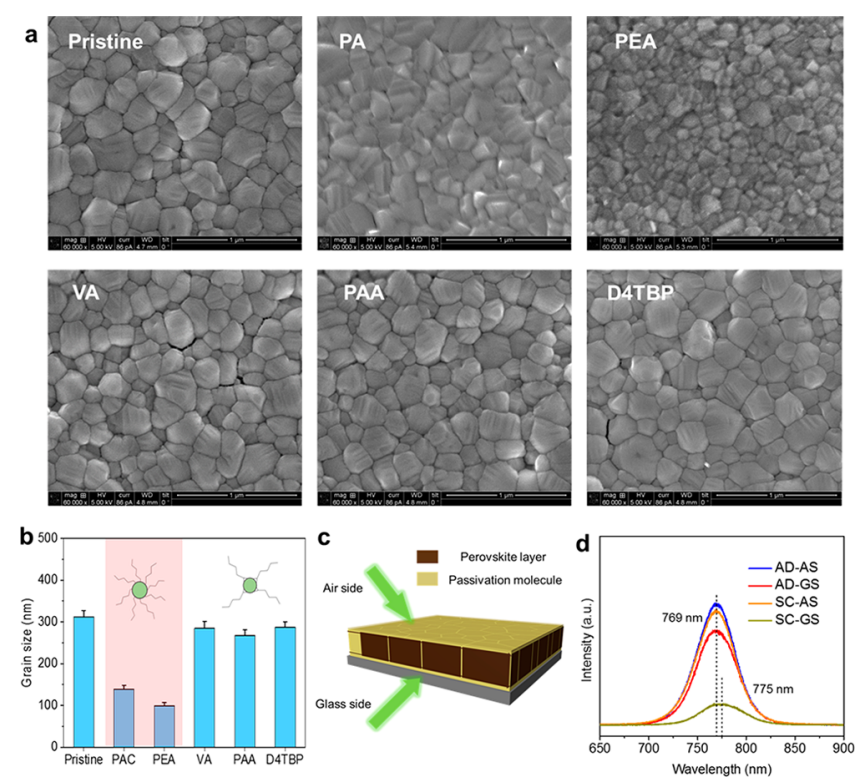

Figure 3. (a) Surface SEM images of perovskite films deposited with different passivation additives. The concentration of all additives is 7.5 $\mathrm{mM}$. (b) Grain size distribution of the perovskite films. Insets show the adsorption of monodentate and bidentate ligands affected by the steric effects. (c) Schematic of the PL measurement of the grain boundary passivated perovskite films. An excitation light of $532 \mathrm{~nm}$ was used to probe the PL property and distribution of passivation molecules. (d) PL spectra of perovskite films with added D4TBP as addictive (AD) and top D4TBP passivation layer by spin-coating (SC) recorded under the excitation light from either air side (AS) or glass side (GS).

the grain boundaries of perovskite when introduced as additives.

We have shown the excellent passivation effect of D4TBP molecules with a prolonged PL lifetime and very low $V_{\mathrm{OC}}$ deficit in solar cells, which could combine the advantages of 4tert-butylphenyl, amine, and carboxyl functional groups. We then optimized the fabrication of perovskite solar cells by using D4TBP as additive to passivate perovskite grains. The concentration of D4TBP in perovskite solution was varied from 2.5 to $10 \mathrm{mM}$. Figure 4a shows the $J-V$ curves of solar cells with varied D4TBP concentration. The maximum PCE increased considerably from $19.7 \%$ to $20.6,20.8$, and $21.4 \%$ for the devices with $2.5,5.0$, and $7.5 \mathrm{mM} \mathrm{D} 4 \mathrm{TBP}$ in perovskite solution, which may be due to more complete wrapping of perovskite grains by D4TBP molecules. The PCE subsequently decreased to $17.4 \%$ at $10 \mathrm{mM}$ D4TBP in perovskite solution (Table S3). Likewise, the average $V_{\mathrm{OC}}$ is $1.08,1.13,1.15,1.20$, and $1.19 \mathrm{~V}$ for D4TBP concentrations of $0,2.5,5.0,7.5$, and $10.0 \mathrm{mM}$, respectively (Figure $4 \mathrm{~b}$ ). No obvious photocurrent hysteresis is identified in these devices (Figure $4 c$ and Table S4). The histogram of the PCEs in Figure S3 shows an average efficiency of $20.55 \pm 0.43 \%$ under simulated AM1.5G illumination. The $J_{S C}$ value obtained from external quantum efficiency (EQE) spectra in Figure S4 is calculated to be 22.1 $\mathrm{mA} \mathrm{cm}{ }^{-2}$, coinciding with the $J-V$ results. The absorption edge determined by the onset of EQE spectra is $\sim 1.57 \mathrm{eV}$ (Figure S5). The champion device was held at maximum power point (MPP) to track the power output. As shown in Figure $4 \mathrm{~d}$, the photocurrent stabilized within seconds to be $20.7 \mathrm{~mA} \mathrm{~cm}^{-2}$ with a PCE of $21.4 \%$. The highest achieved $V_{\mathrm{OC}}$ in these devices reaches $1.23 \mathrm{~V}$, with a PCE of $21.12 \%$ (Figure 

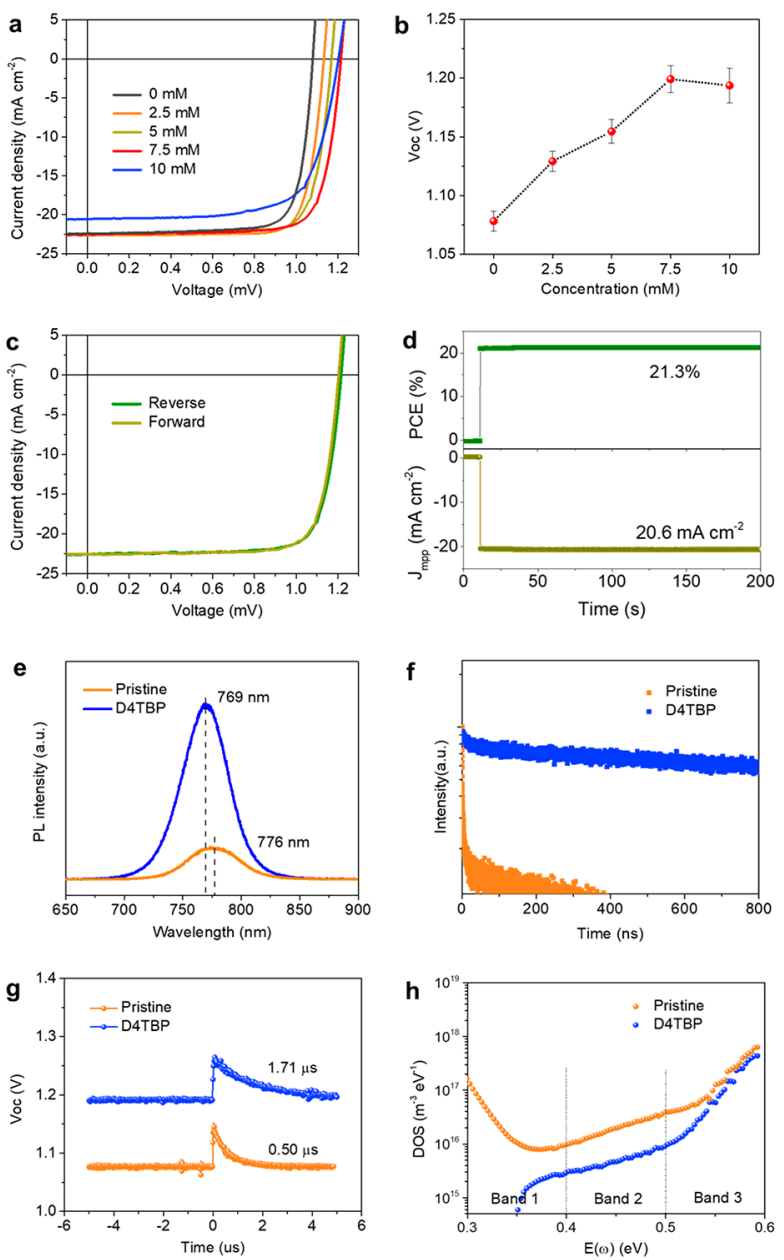

Figure 4. (a) $J-V$ characteristics and (b) $V_{\mathrm{OC}}$ distribution of the perovskite solar cells with different concentrations of D4TBP in perovskite precursor solution. (c) $J-V$ curves of the champion perovskite solar cell with D4TBP passivation with forward and reverse scan. (d) Steady-state measurement of $J_{\mathrm{SC}}$ and PCE of the champion D4TBP perovskite solar cells. (e) PL and (f) TRPL spectra of pristine and D4TBP perovskite films. (g) TPV curves and (h) trap density in the pristine and D4TBP perovskite devices. D4TBP is added as additive to passivate the perovskites film and devices.

S6a). The $V_{\text {OC }}$ was confirmed by measuring the stabilized $V_{\text {OC, }}$ as shown in Figure $\mathrm{S} 6 \mathrm{~b}$. The $V_{\mathrm{OC}}$ loss is thus only $0.34 \mathrm{~V}$, which is the lowest $V_{\mathrm{OC}}$ loss reported, implying a very effective defect passivation of perovskite surface and grain boundaries.

The good passivation effect of D4TBP was also evidenced by the steady-state PL spectra that D4TBP sample possess $\sim 5.7$ times stronger PL emission with about $7 \mathrm{~nm}$ blue-shift compared with the pristine sample (Figure 4e). Furthermore, the PL lifetime of the D4TBP sample is further elongated so that the $\tau_{1}$ and $\tau_{2}$ improved to be 17.71 and $1280.71 \mathrm{~ns}$, respectively, which is even longer than the spin-casted sample (Figure 4f). Devices were then irradiated under AM 1.5G simulated illumination and laser pulses $(337 \mathrm{~nm}, 4 \mathrm{~ns})$ to measure the decay of transient photovoltage signals. As seen in Figure $4 \mathrm{~g}$, the charge-recombination lifetime was increased from $0.50 \mu \mathrm{s}$ in the pristine device to $1.71 \mu \mathrm{s}$ for D4TBPtreated device. As a well-established method in thin film photovoltaics, thermal admittance spectroscopy was operated to measure the trap density of states (tDOS) of the control and D4TBP devices. Figure $4 \mathrm{~h}$ describes that the D4TBP device had the lower tDOS almost over the whole trap depth region. The density of shallower trap states $(0.3-0.5 \mathrm{eV})$ of D4TBP device is about 1 order of magnitude lower than that of the control device. Our previous characterizations have disclosed that the shallow traps are mainly located at the grain boundaries. $^{22}$ We therefore infer that the trap states are effectively reduced upon the D4TBP passivation. We have shown the addictive route can significantly reduce the density of trap states and enhance the PL intensities of perovskite films, with apparent better photovoltaic parameters compared with that based on spin-coating strategy. In fact, the spatial distribution of D4TBP molecules would be dependent on the chemical methods when incorporated. The D4TBP addictive is thought to be enriched at grain boundaries as evidenced by their independence of high PL intensities on both sides, whereas the film passivated by spin-coating only passivates the top side. The good molecular passivation effect of grain boundaries, other than only top surface, explains the extreme low $V_{\mathrm{OC}}$ deficit of $0.34 \mathrm{~V}$ of the passivated device.

\section{CONCLUSION}

In summary, we demonstrated a simple, generic strategy to $\mathrm{p}$-i$\mathrm{n}$ structure solar cells the electronic defects of the hybrid perovskite films. Multiple kinds of defects, such as the ionic or neutral ones, have been addressed based on the ionic and coordination interactions with certain molecular structures (Figure 5). We further enabled the grain boundary passivation

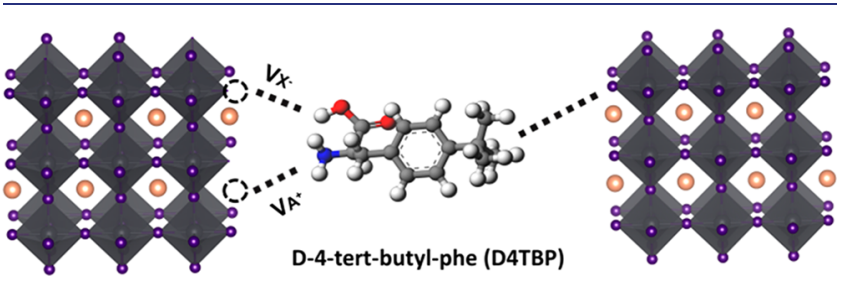

Figure 5. Schematic illustration of the origin of D4TBP passivation effect on different defect sites.

by controlling the structure of small molecules. The defect passivation reduces the $V_{\mathrm{OC}}$ deficit of the $\mathrm{p}-\mathrm{i}-\mathrm{n}$-structured device to $0.34 \mathrm{~V}$ and boosts the efficiency to $21.4 \%$. Our finding provides new guidelines for surface molecular modulation of hybrid perovskites.

\section{EXPERIMENTAL SECTION}

Device Fabrication. Patterned ITO glass substrates were first cleaned by ultrasonication with soap, acetone, and isopropanol. The hole transport layer poly(bis(4-phenyl)(2,4,6-trimethylphenyl)amine) (PTAA) with a concentration of $2 \mathrm{mg} \mathrm{mL}^{-1}$ dissolved in toluene was spin-coated at the speed of $4000 \mathrm{rpm}$ for $35 \mathrm{~s}$ and then annealed at $100{ }^{\circ} \mathrm{C}$ for $10 \mathrm{~min}$. Before depositing perovskite films, the PTAA film was prewetted by spinning $80 \mu \mathrm{L}$ of DMF at $4000 \mathrm{rpm}$ for $15 \mathrm{~s}$ to improve the wetting property of the perovskite precursor solution. The perovskite precursor solution composed of mixed cations (lead $(\mathrm{Pb})$, cesium $(\mathrm{Cs})$, formamidinium (FA), and methylammonium (MA)) and halides (I and $\mathrm{Br}$ ) was dissolved in mixed solvent $(\mathrm{DMF} / \mathrm{DMSO}=4: 1)$ with a chemical formula of $\mathrm{Cs}_{0.05} \mathrm{FA}_{0.81}{ }^{-}$ $\mathrm{MA}_{0.14} \mathrm{PbI}_{2.55} \mathrm{Br}_{0.45}{ }^{13}$ Then $80 \mu \mathrm{L}$ of precursor solution was spun onto PTAA at $2000 \mathrm{rpm}$ for $2 \mathrm{~s}$ and $4000 \mathrm{rpm}$ for $20 \mathrm{~s}$, and the film was quickly washed with $130 \mu \mathrm{L}$ of toluene at $18 \mathrm{~s}$ during spin-coating. Subsequently, the sample was annealed at $65{ }^{\circ} \mathrm{C}$ for $10 \mathrm{~min}$ and 100 ${ }^{\circ} \mathrm{C}$ for $10 \mathrm{~min}$. For the surface passivation, the passivation molecules were dissolved in isopropanol with the concentration of $1 \mathrm{mg} / \mathrm{mL}$ and was spun at $4000 \mathrm{rpm}$ for $30 \mathrm{~s}$. The films were then annealed at 100 
${ }^{\circ} \mathrm{C}$ for $10 \mathrm{~min}$. For the grain boundary passivation, molecules were directly added into the perovskite precursor as additive with the concentration between 2.5 and $10 \mathrm{mM}$. Hydroiodic acid (HI, $57 \mathrm{wt} \%$ in water) was added by equal molar ratio with the passivation molecules in all samples. The devices were finished by thermally evaporating C60 $(30 \mathrm{~nm}), \mathrm{BCP}(8 \mathrm{~nm})$, and copper $(140 \mathrm{~nm})$ in sequential order.

Characterization. Crystallographic information for the assynthesized crystals was obtained by a Rigaku D/Max-B X-ray diffractometer with Bragg-Brentano parafocusing geometry, a diffracted beam monochromator, and a conventional cobalt target $\mathrm{X}$-ray tube set to $40 \mathrm{kV}$ and $30 \mathrm{~mA}$. The morphology and structure of the samples were characterized by Quanta 200 FEG environmental scanning electron microscope. Optical absorption spectra were measured by means of an Evolution 201/220 UV/vis spectrophotometer. Time-resolved photoluminescence (TRPL) was performed on the perovskite films grown on varied substrates by a Horiba DeltaPro fluorescence lifetime system, which equipped with a DeltaDiode (DD-405) pulse laser diode with wavelength of 404 $\mathrm{nm}$. The laser excitation energy in the measurement was $20 \mathrm{pJ}$ pulse $^{-1}$. The PL spectrum was recorded by a iHR320 photoluminescence spectrometer at room temperature. The excitation source for PL measurement is a $532 \mathrm{~nm}$ green laser with an intensity of $10 \mathrm{~mW} \mathrm{~cm}{ }^{-2}$ from Laserglow Technologies. The sample for PL and TRPL measurements is perovskite layer on glass. The $J-V$ analysis of solar cells was performed using a solar light simulator (Oriel 67005, $150 \mathrm{~W}$ ), and the power of the simulated light was calibrated to 100 $\mathrm{mW} \mathrm{cm}^{-2}$ with a silicon (Si) diode (Hamamatsu S1133) equipped with a Schott visible-color glass filter (KG5). All cells were measured using a Keithley 2400 source meter with scan rate of $0.1 \mathrm{~V} \mathrm{~s}^{-1}$. The steady-state PCE was measured by monitoring current with the largest power output bias voltage and recording the value of the photocurrent. External quantum efficiency curves were characterized with a Newport QE measurement kit by focusing a monochromatic beam of light onto the devices. The tDOS of solar cells was derived from the frequency-dependent capacitance $(C-f)$ and voltagedependent capacitance $(C-V)$, which were obtained from the thermal admittance spectroscopy (TAS) measurement performed by a LCR meter (Agilent E4980A). The transient photovoltage was measured under 1 sun illumination. An attenuated UV laser pulse (SRS NL 100 nitrogen laser) was used as a small perturbation to the background illumination on the device. The laser-pulse-induced photovoltage variation and the $V_{\mathrm{OC}}$ are produced by the background illumination. The wavelength of the $\mathrm{N}_{2}$ laser was $337 \mathrm{~nm}$, the repeating frequency was $\sim 10 \mathrm{~Hz}$, and the pulse width was $<3.5 \mathrm{~ns}$.

\section{ASSOCIATED CONTENT}

\section{Supporting Information}

The Supporting Information is available free of charge on the ACS Publications website at DOI: 10.1021/jacs.8b13091.

Additional XRD patterns, SEM images, PCE distribution, EQE spectra, PL lifetimes and photovoltaic performances of the devices (PDF)

\section{AUTHOR INFORMATION}

\section{Corresponding Author}

*E-mail: jhuang@unc.edu.

\section{ORCID $\odot$}

Shuang Yang: 0000-0002-8244-3002

Xun Xiao: 0000-0002-9810-2448

Xiao Cheng Zeng: 0000-0003-4672-8585

Jinsong Huang: 0000-0002-0509-8778

Notes

The authors declare no competing financial interest.

\section{ACKNOWLEDGMENTS}

This work is financially supported by Air Force Office of Scientific Research (AFOSR) (Grant A9550-16-1-0299) and National Science Foundation under Award DMR-1420645.

\section{REFERENCES}

(1) National Renewable Energy Laboratory, Best research-cell efficiencies chart; https://www.nrel.gov/pv/assets/pdfs/pvefficiencies-07-17-2018.pdf.

(2) Zhou, H.; Chen, Q.; Li, G.; Luo, S.; Song, T.; Duan, H.-S.; Hong, Z.; You, J.; Liu, Y.; Yang, Y. Interface engineering of highly efficient perovskite solar cells. Science 2014, 345, 542-546.

(3) Shin, S. S.; Yeom, E. J.; Yang, W. S.; Hur, S.; Kim, M. G.; Im, J.; Seo, J.; Noh, J. H.; Seok, S. I. Colloidally prepared La-doped $\mathrm{BaSnO}_{3}$ electrodes for efficient, photostable perovskite solar cells. Science 2017, 356, 167-171.

(4) Saliba, M.; Matsui, T.; Domanski, K.; Seo, J.-Y.; Ummadisingu, A.; Zakeeruddin, S. M.; Correa-Baena, J.-P.; Tress, W. R.; Abate, A.; Hagfeldt, A.; Grätzel, M. Incorporation of rubidium cations into perovskite solar cells improves photovoltaic performance. Science 2016, 354, 206-209.

(5) Chen, W.; Wu, Y.; Yue, Y.; Liu, J.; Zhang, W.; Yang, X.; Chen, H.; Bi, E.; Ashraful, I.; Grätzel, M.; Han, L. Efficient and stable largearea perovskite solar cells with inorganic charge extraction layers. Science 2015, 350, 944-948.

(6) Yang, S.; Wang, Y.; Liu, P.; Cheng, Y.-B.; Zhao, H. J.; Yang, H. G. Functionalization of perovskite thin films with moisture-tolerant molecules. Nat. Energy 2016, 1, 15016.

(7) Yin, W.-J.; Shi, T.; Yan, Y. Unusual defect physics in $\mathrm{CH}_{3} \mathrm{NH}_{3} \mathrm{PbI}_{3}$ perovskite solar cell absorber. Appl. Phys. Lett. 2014, $104,063903$.

(8) Dong, Q.; Fang, Y.; Shao, Y.; Mulligan, P.; Qiu, J.; Cao, L.; Huang, J. Electron-hole diffusion lengths $>175 \mu \mathrm{m}$ in solution-grown $\mathrm{CH}_{3} \mathrm{NH}_{3} \mathrm{PbI}_{3}$ single crystals. Science 2015, 347, 967-970.

(9) de Quilettes, D. W.; Vorpahl, S. M.; Stranks, S. D.; Nagaoka, H.; Eperon, G. E.; Ziffer, M. E.; Snaith, H. J.; Ginger, D. S. Impact of microstructure on local carrier lifetime in perovskite solar cells. Science 2015, 348, 683-686.

(10) Zheng, X.; Chen, B.; Dai, J.; Fang, Y.; Bai, Y.; Lin, Y.; Wei, H.; Zeng, X. C.; Huang, J. Defect passivation in hybrid perovskite solar cells using quaternary ammonium halide anions and cations. Nat. Energy 2017, 2, 17102.

(11) Chen, Q.; Zhou, H.; Song, T.-B.; Luo, S.; Hong, Z.; Duan, H. S.; Dou, L.; Liu, Y.; Yang, Y. Controllable self-induced passivation of hybrid lead iodide perovskites toward high performance solar cells. Nano Lett. 2014, 14, 4158-4163.

(12) Shao, Y.; Xiao, Z.; Bi, C.; Yuan, Y.; Huang, J. Origin and elimination of photocurrent hysteresis by fullerene passivation in $\mathrm{CH}_{3} \mathrm{NH}_{3} \mathrm{PbI}_{3}$ planar heterojunction solar cells. Nat. Commun. 2014, 5,5784 .

(13) Xu, J.; Buin, A.; Ip, A. H.; Li, W.; Voznyy, O.; Comin, R.; Yuan, M.; Jeon, S.; Ning, Z.; McDowell, J. J.; Kanjanaboos, P.; Sun, J.-P.; Lan, X.; Quan, L. N.; Kim, D. H.; Hill, I. G.; Maksymovych, P.; Sargent, E. H. Perovskite-fullerene hybrid materials suppress hysteresis in planar diodes. Nat. Commun. 2015, 6, 7081.

(14) Braly, I. L.; deQuilettes, D. W.; Pazos-Outón, L. M.; Burke, S.; Ziffer, M. E.; Ginger, D. S.; Hillhouse, H. W. Hybrid perovskite films approaching the radiative limit with over $90 \%$ photoluminescence quantum efficiency. Nat. Photonics 2018, 12, 355-361.

(15) Tan, H.; Jain, A.; Voznyy, O.; Lan, X.; de Arquer, F. P. G.; Fan, J. Z.; Quintero-Bermudez, R.; Yuan, M.; Zhang, B.; Zhao, Y.; Fan, F.; Li, P.; Quan, L. N.; Zhao, Y.; Lu, Z.-H.; Yang, Z.; Hoogland, S.; Sargent, E. H. Efficient and stable solution-processed planar perovskite solar cells via contact passivation. Science 2017, 355, $722-726$.

(16) Rajagopal, A.; Stoddard, R. J.; Jo, S. B.; Hillhouse, H. W.; Jen, A. K.-Y. Overcoming the photovoltage plateau in large bandgap perovskite photovoltaics. Nano Lett. 2018, 18, 3985-3993. 
(17) Walsh, A.; Scanlon, D. O.; Chen, S.; Gong, X.; Wei, S. H. Selfregulation mechanism for charged point defects in hybrid halide perovskites. Angew. Chem., Int. Ed. 2015, 54, 1791-1794.

(18) Liu, Y.; Palotas, K.; Yuan, X.; Hou, T.; Lin, H.; Li, Y.; Lee, S.-T. Atomistic origins of surface defects in $\mathrm{CH}_{3} \mathrm{NH}_{3} \mathrm{PbBr}_{3}$ perovskite and their electronic structures. ACS Nano 2017, 11, 2060-2065.

(19) Maiberg, M.; Hölscher, T.; Zahedi-Azad, S.; Scheer, R. Theoretical study of time-resolved luminescence in semiconductors. III. Trap states in the band gap. J. Appl. Phys. 2015, 118, 105701.

(20) Protesescu, L.; Yakunin, S.; Bodnarchuk, M. I.; Krieg, F.; Caputo, R.; Hendon, C. H.; Yang, R. X.; Walsh, A.; Kovalenko, M. V. Nanocrystals of cesium lead halide perovskites $\left(\mathrm{CsPbX}_{3}, \mathrm{X}=\mathrm{Cl}, \mathrm{Br}\right.$, and I): novel optoelectronic materials showing bright emission with wide color gamut. Nano Lett. 2015, 15, 3692-3696.

(21) De Roo, J. D.; Ibáñez, M.; Geiregat, P.; Nedelcu, G.; Walravens, W.; Maes, J.; Martins, J. C.; Van Driessche, I. V.; Kovalenko, M. V.; Hens, Z. Highly dynamic ligand binding and light absorption coefficient of cesium lead bromide perovskite nanocrystals. ACS Nano 2016, 10, 2071-2081.

(22) Wang, F.; Geng, W.; Zhou, Y.; Fang, H.-H.; Tong, C.-J.; Loi, M. A.; Liu, L.-M.; Zhao, N. Phenylalkylamine passivation of organolead halide perovskites enabling high-efficiency and air-stable photovoltaic cells. Adv. Mater. 2016, 28, 9986-9992.

(23) Lindblad, R.; Bi, D.; Park, B.-W.; Oscarsson, J.; Gorgoi, M.; Siegbahn, H.; Odelius, M.; Johansson, E. M. J.; Rensmo, H. Electronic structure of $\mathrm{TiO}_{2} / \mathrm{CH}_{3} \mathrm{NH}_{3} \mathrm{PbI}_{3}$ perovskite solar cell interfaces. J. Phys. Chem. Lett. 2014, 5, 648-653.

(24) Bi, D.; Yi, C.; Luo, J.; Décoppet, J.-D.; Zhang, F.; Zakeeruddin, S. M.; Li, X.; Hagfeldt, A.; Grätzel, M. Polymer-templated nucleation and crystal growth of perovskite films for solar cells with efficiency greater than 21\%. Nat. Energy 2016, 1, 16142.

(25) Niu, G.; Li, We.; Meng, F.; Wang, L.; Dong, H.; Qiu, Y. Study on the stability of $\mathrm{CH}_{3} \mathrm{NH}_{3} \mathrm{PbI}_{3}$ films and the effect of postmodification by aluminum oxide in all-solid-state hybrid solar cells. $J$. Mater. Chem. A 2014, 2, 705-710.

(26) Buin, A.; Pietsch, P.; Xu, J.; Voznyy, O.; Ip, A. H.; Comin, R.; Sargent, E. H. Materials processing routes to trap-free halide perovskites. Nano Lett. 2014, 14, 6281-6286.

(27) Wang, S.; Jiang, Y.; Juarez-Perez, E. J.; Ono, L. K.; Qi, Y. Accelerated degradation of methylammonium lead iodide perovskites induced by exposure to iodine vapour. Nat. Energy 2017, 2, 16195.

(28) Zhang, W.; Pathak, S.; Sakai, N.; Stergiopoulos, T.; Nayak, P. K.; Noel, N. K.; Haghighirad, A. A.; Burlakov, V. M.; deQuilettes, D. W.; Sadhanala, A.; Wang, W.; Li, L.; Ginger, D. S.; Friend, R. H.; Snaith, H. J. Enhanced optoelectronic quality of perovskite thin films with hypophosphorous acid for planar heterojunction solar cells. Nat. Commun. 2015, 6, 10030.

(29) Liu, Z.-B.; Tian, J.-G.; Zang, W.-P.; Zhou, W.-Y.; Song, F.; Zhang, C.-P.; Zheng, J.-Y.; Xu, H. Flexible alteration of optical nonlinearities of iodine charge-transfer complexes in solutions. Opt. Lett. 2004, 29, 1099-1101.

(30) Takeuchi, H.; Omogo, B.; Heyes, C. D. Are bidentate ligands really better than monodentate ligands for nanoparticles? Nano Lett. 2013, 13, 4746-4752. 